\title{
Researches on mechanical vibrations used in assistive devices manufacturing
}

\author{
Răzvan Bogățeanu ${ }^{1,}$, , Claudiu Bîșu ${ }^{2}$, Constantin Dogariu ${ }^{2}$, Sorin Mihai Croitoru ${ }^{2}$, and Dana \\ Tilină ${ }^{2}$ \\ ${ }^{1}$ University Politehnica of Bucharest, Doctoral School Engineering and Management of \\ Technological Systems, 313 Spl. Independentei, 060042, Bucharest, Romania \\ ${ }^{2}$ University Politehnica of Bucharest, Faculty of Engineering and Management of Technological \\ Systems, Robotics and Production Systems Department, 313 Spl. Independentei, 060042, Romania
}

\begin{abstract}
Deformable structures vibrate (oscillatory motion) under the action of impulses or variable forces. Vibration of a mechanical structure results from the transformation of potential energy into kinetic energy and could be used in the construction of assistive devices. The vibratory devices for posttraumatic rehabilitation of upper limbs can stimulate muscular, circulatory and neurological systems. The paper presents the necessary elements for the personalization of rehabilitation devices like orthoses and some theoretical and experimental results related to this subject. For the persons with disabilities, the integration of enabling technologies and attributes embracing mobility, sensors and actuators provides the possibility of achieving independence in their social life. The paper underlines the importance of the systematic application of technologies, engineering methodologies or other scientific principles used in order to overpass the barriers confronted by people with disabilities.
\end{abstract}

\section{Introduction}

Vibrotherapy is used in rehabilitation of persons who suffer from motor impairments and in the design of assistive therapy devices. The basic principle of vibrotherapy consists in finding a stimulation frequency that will improve the patient's health condition. There are several vibration sources that can be used to achieve a broad spectrum of amplitude and vibration. One of them is the electricity, which provides electrical impulses [1]. In many medical situations the use of electrical impulses is difficult without a major impact on improving health.

In this regard, the present paper proposes a vibrotherapy based method which would enable a steady and continuous recovery, in such a way that the patient benefits from a complementary passive recovery method at home, as well as in the medical recovery clinic [2]. Depending on the existing disease and the patient's health condition the vibration wave is calibrated according to biomechanical parameters, determining the amplitude, frequency and usages cycles [3].

\footnotetext{
* Corresponding author: razvan.bogateanu@gmail.com
} 
The paper focuses on the use of vibrotherapy for upper limbs disorders. This would mean designing and manufacturing an orthosis specifically tailored to the needs of the affected limb, which is easily and safely operated and delivers the desired results [4].

Although there is a vast and complex assortment of equipment and devices used in the recovery of upper limbs, there is a need for personalized and financially accessible devices, which should be easy to handle and wear, and would allow patients to extend their treatment and recommended physical exercise to their homes [5 - 6]. This would greatly reduce the recovery period and would speed up the reintegration of patients [7].

Modern-day medicine comprises three interconnected branches with specific and shared elements: preventive, curative and rehabilitative medicine. In the past, curative medicine was given a great importance [5]. The physician's task was to determine a diagnosis and to apply a specific treatment. In the present, however, preventive medicine and rehabilitative medicine have a great deal of importance [1-2].

Rehabilitative care is a medical and social activity which has lately registered a significant technological and academic progress in its pursuit of socially and professionally reintegrating patients going through a recovery period [7]. Assistive devices were developed, especially orthoses and prostheses, for facilitating recovery and rehabilitation [7 - 9].

In this paper we perform a dynamic characterisation of a vibratory orthosis for the recovery of upper limbs after a trauma. The vibratory orthosis is the subject of a patent application. The novelty of this research is the integration of parametrical vibrations waves generated by micro-motors into a specific design orthosis. As it is known from literature and medical experience, the use of vibrotherapy simulates and improves motricity [10 - 11]. But the use of vibrations waves with permanent assistance of amplitude and frequency parameters and their application based on resonance frequencies represents a major improvement in recovery time.

\section{Vibrotherapy for stimulating the muscle strength and rehabilitation}

Like electrotherapy, there is recovery and rehabilitation therapy based on the use of vibration field, also called vibrotherapy, making a bridge the mechanotherapy and medicine field. It is a technique used since 1860 in various medical cases, even by Dr. Jonas Gustav Zander. But which over the past 30 years has seen a significant development, relying on new assistive equipment technologies. At the beginning of this appearance, vibrotherapy was applied specially to joints and neuromuscular disorders.

After Dr. Jonas Gustav Wilhelm Zander exist 4 classes of mechanotherapy: active motion devices for upper and lower limbs, torso and balancing movements; passive motion devices for kinetic applications; devices for all types of mechanical massage, using vibrations, tapping, kneading, stroking; orthopaedic devices for passive and active recovery, as well as a few devices for taking scoliosis measurements. Actually, the vibrotherapy is now widely accepted in the fields of physiotherapy, rehabilitation and sport medicine for the treatment of different stages of illnesses and injuries.

The Ivan Uher shown that the mechanical oscillatory-cycloid vibrations applied to the muscle belly or tendons can stimulate sensory receptors. Also, the effect of vibration depends on the proprieties of the muscle, vibration frequency, level of pre-contraction, position of the body, etc. [12]. As long as exposure to vibration is known to lead to serious illness (eg. truck drivers and tractors, special machine operators, etc.), the use of vibration in medical field leads to extremely high results beneficial for recovery. Knowing the specific properties of the stimuli leads to an optimal use of the assistive equipment so as to obtain the maximum operating conditions in frequency and amplitude. Recent researches 
have been reports that the transmission of vibratory stimuli throughout responses which depend on the proprieties of these stimuli. Vibration generated by a platform and transmitted to the body, activates a series of sensory receptors, from cutaneous to muscular [11].

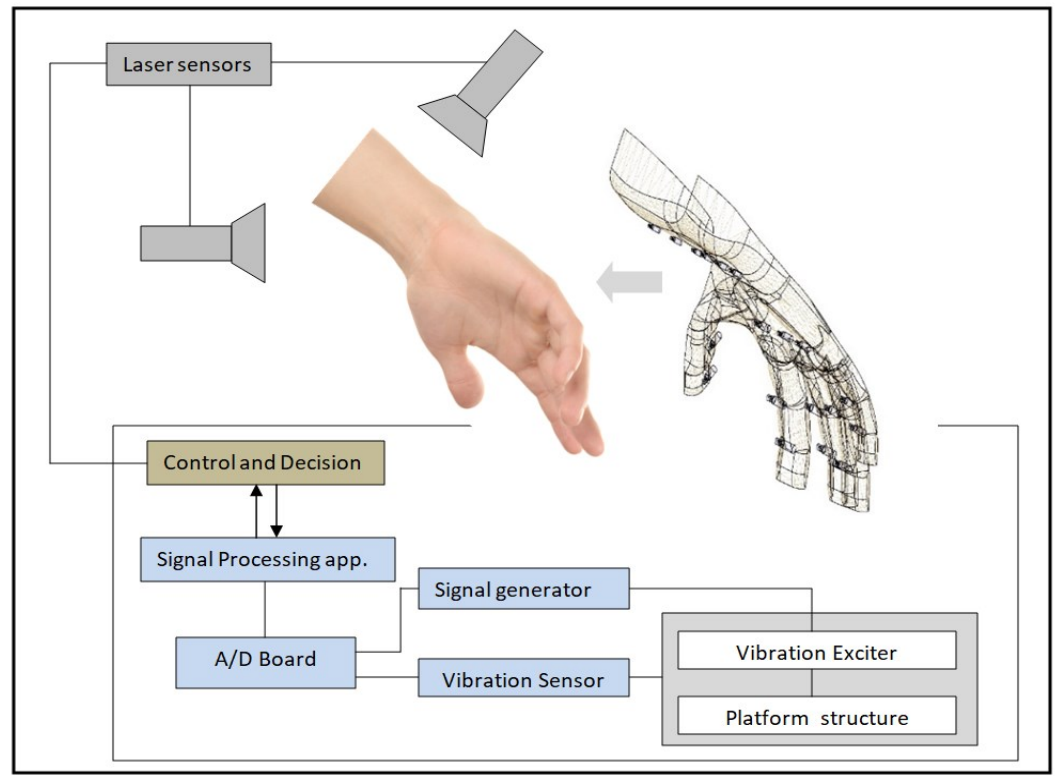

Fig. 1. Close loop for vibratory orthosis.

Vibration is generated by micro-motors and controlled by frequency via a signal generator. For an optimal frequency, the natural frequency of the upper limb is measured, depending on the patient's condition and the orthotic will generate vibration within the frequency proper range.

This paper proposes an orthosis in respect to biomechanical principles, for patient's rehabilitation at home [13]. This orthosis is personalized and allows the possibility to adjust the frequency depending on the necessities and medical condition of the patient. Up to present, many assistive devices for upper lamb illness used electrical impulses application. Frequency is adjusted to the necessities of the patient who must be rehabilitated.

\section{Experimental procedures}

For an in-depth knowledge of the orthosis an experimental procedure has been designed so that it can be evaluated qualitatively. The orthosis was made in house with own additive technology, for the time being is subject to protection conditions following the patent application procedure. In this research the orthosis was subjected to the action of the vibrations generated in the open configuration (free case) but also in the closed configuration, fixed directly on the hand.

The orthosis vibrations are produced by several micro-motors, powered at a voltage of $3 \mathrm{~V}$, circuit being programmed depending on the specific patient case. Parameters to be adjusted may be: functioning duration, intensity, type of vibration (ascendant, descendent, low frequency, high frequency etc.).

Basic element to more rapid hand stimulation and innervation is a special designed and manufactured device, as seen in figure 2 . 


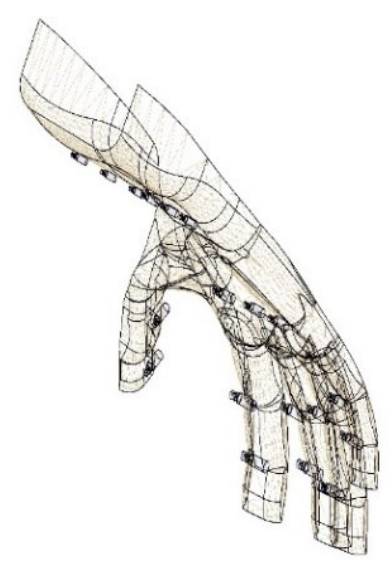

Fig. 2. Orthosis CAD model.

For orthoses characterization there was realized an experimental study regarding frequencies and amplitudes of vibrations generated by all micro-motors positioned onto the orthoses. Determination of frequency range was made in two cases: self-excited (free case) and dynamic. In the last case the orthoses were put on patient's hand.

In natural case (self-excited) the orthosis was put on a test rig, without fixture and without stiffening. Frequency range was determined by means of a Bruel\&Kjaer accelerometer, having sensitivity of $100 \mathrm{mV} / \mathrm{g}$. Vibration signal was acquisitioned and processed by a National Instruments USB 4431 module (Fig. 3).

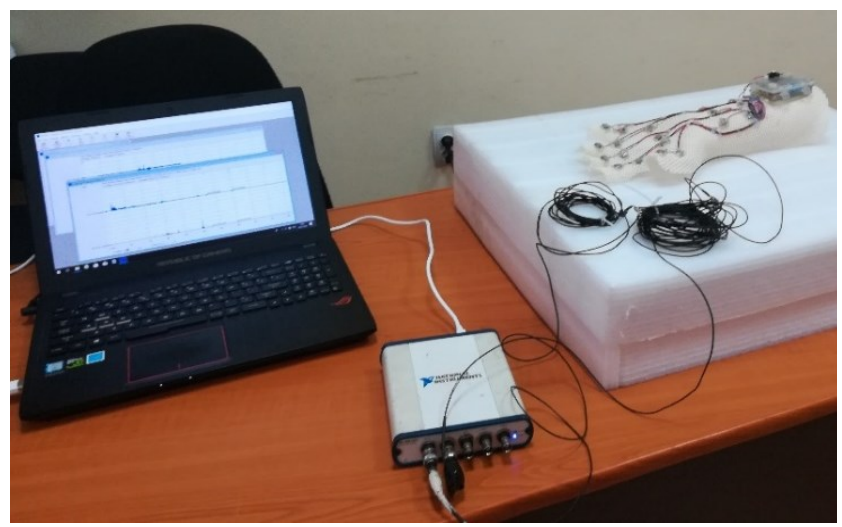

Fig. 3. Frequency measurement in self excited case.

Contact surface of the orthosis was divided in 18 representative elements. For each element frequency and amplitude were measured. The study objective was to highlight the frequency range generated by the orthosis and adequate it to the accepted by the human body values.

Stimulation of the muscles will generate effects onto the circulatory and neurologic systems.

From circulatory system point of view, in case of an upper limb paresis, at frequencies less than $50 \mathrm{~Hz}$, consecutive vasoconstriction will give also a contraction at neurologic level. This command will be recalled onto the cortex, being possible to transmit a nervous impulse finalized with a voluntary contraction.

In case of spastic plegia treatment vibrations having frequencies more than $50 \mathrm{~Hz}$ will determine a relaxant effect. 
After modification of the applied voltage it can be obtained a benefit value for the patient, depending on his resistivity [14].

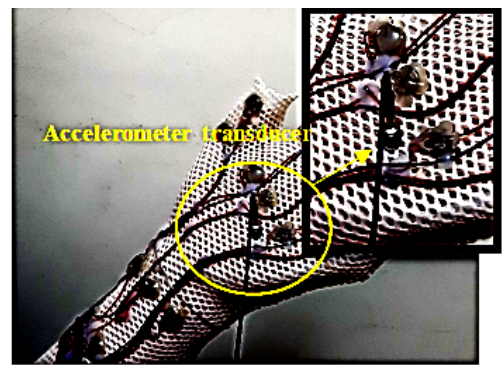

Fig. 4. Measurement in reel case.

Relating the dynamic case measurement was done fixing the orthosis on the hand, obtaining the rigidity of the orthosis structure, vibrations being highlighted by means of and accelerometer positioned in every and each of the 18 measuring points.

Experimental protocol allowed highlights the frequency and amplitude ranges for the two studied cases, leading to obtaining the signals in time-domain and frequency-domain.

\section{Results and discussion}

The connection between neurons is chemical in nature and the nature of information exchanged between the neurons is electrical. This information can be stimulated through an electrical current by forcing an electrical current of higher intensity on the same informational pathway. Although they are regarded as relaxation products, most vibration devices available on the market can be adapted, through small alterations, to be used in the recovery of the locomotor system [15].

By analysing only a small fraction of the upper limbs recovery assistive equipment on the market, the importance given to their manufacture around the world becomes evident. It has been observed that there is a broad variety of equipment, systems and devices from the most basic designs to the most complex. They target specific issues or more general ones in the neuromotor recovery of upper limbs, statically or dynamically, osteoarticular or muscular.

This is a practical, comfortable and easy to use new concept that is readily accessible to patients and allows a more efficient recovery, given the fact that this orthosis can be used at home, as a continuation of the therapy performed in the presence of a physician or kinetotherapist, and it can quickly improve the quality of life.

Figure 5 shows the waveform signal in the self-excited case, where the structure is excited by micro-motors having a excited frequency around $100 \mathrm{~Hz}$. The orthosis was tested in the free case to observe the behaviour of its structure and the main vibration mode. The amplitudes of the vibrations are amplified by the dynamic system of the orthosis, allowing a characterisation of the structure in terms of stiffness and damping. 


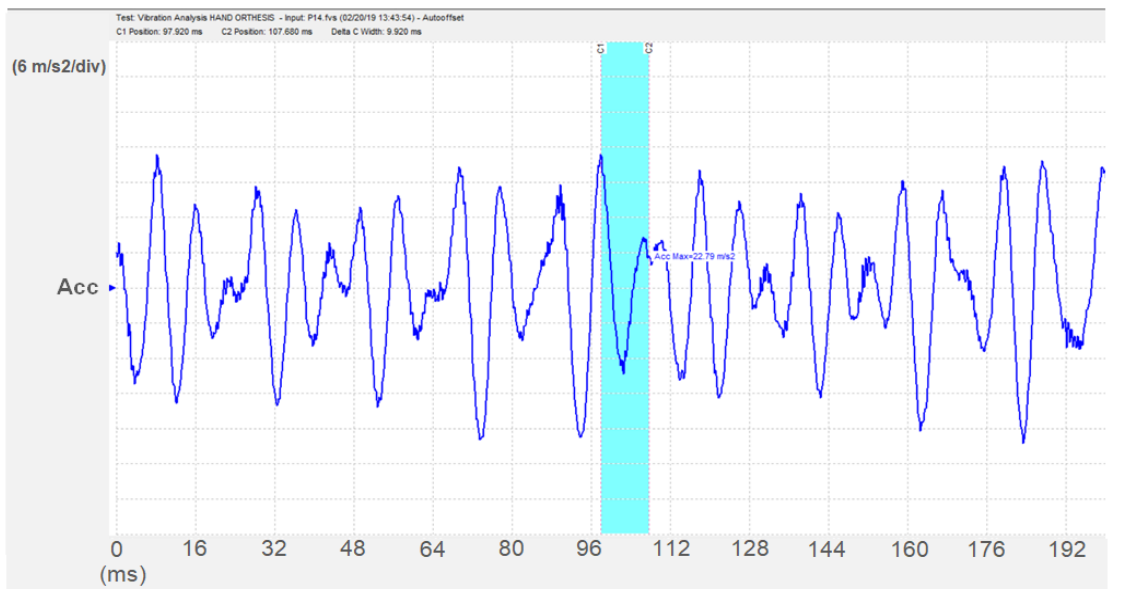

Fig. 5. Waveform diagram in self excited case.

In frequency domain vibrations (fig. 6) are around the fundamental frequency of 100 $\mathrm{Hz}$. A result of the structure of the orthosis and propagation of the vibrations there are also present the low frequencies (LF) in the $45-70 \mathrm{~Hz}$ range, but also a higher frequency (HF) component around $150 \mathrm{~Hz}$. The $45-70 \mathrm{~Hz}$ is one of the frequency ranges of interest for the orthosis, there are many health rehabilitation applications within this frequency range.

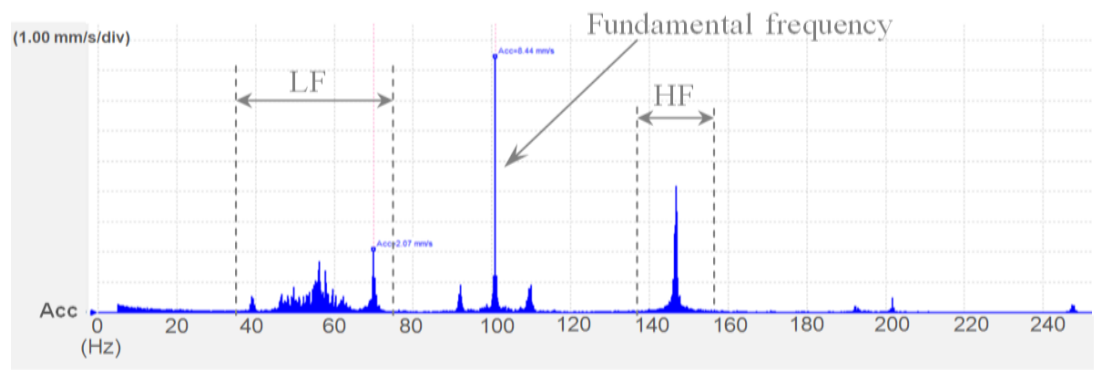

Fig. 6. Spectrum frequency in self excited case.

The same dynamic behaviour is also present in the situation of using the orthosis directly on the hand, in the real case (fig .7). The waveform has the same characteristic of the signal, the equivalent frequency period of $100 \mathrm{~Hz}$. The shape of the time wave signal in the real case is similar as in the free excitation test [15]. The amplitude of the vibrations shows variations because of the contact between the hand and the orthosis.

In the frequency spectrum during the orthosis test on the hand (fig.8) the fundamental frequency $100 \mathrm{~Hz}$ is the central component, with the high energy and represent the carrier frequency with side bands. The orthosis generates the vibration also in the LF range, where many phases of nerve excitation are applied during the recovery period. There are also medical conditions when oscillations must take place at much higher frequencies reaching $100 \mathrm{~Hz}$ or more, for a more severe excitement of poor nerves. In order to use a variable frequency range according to the medical need, the orthosis will be equipped with a frequency changer. For this purpose, an analysis of the orthosis behaviour was made considering a fixed frequency. 


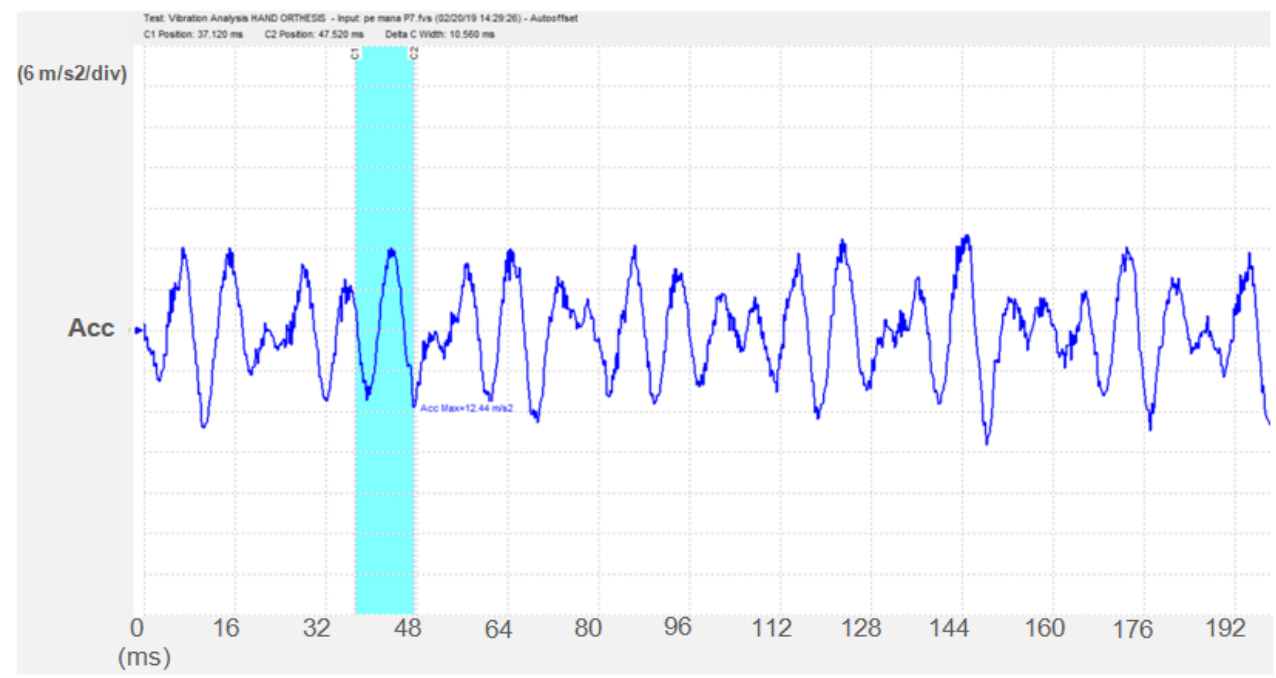

Fig. 7. Waveform diagram in reel case.

There are areas of contact where the stiffness is higher by firm contact between the hand and the surface of the orthosis, changing both the frequency and amplitude. In the figure 9 the fundamental frequency increase reaching up to $110 \mathrm{~Hz}$, as a result of stiffening.

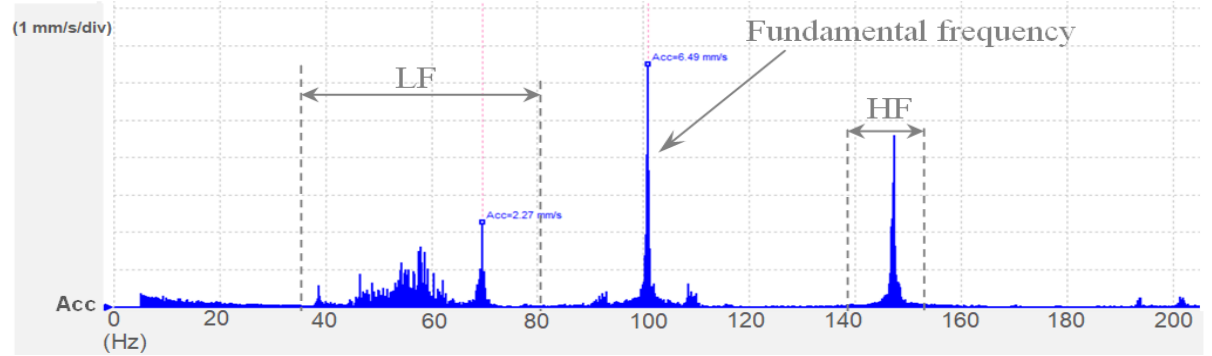

Fig. 8. Spectrum frequency in self excited case.

The orthosis structure configuration is stable and able to be applied in the various dynamic conditions.

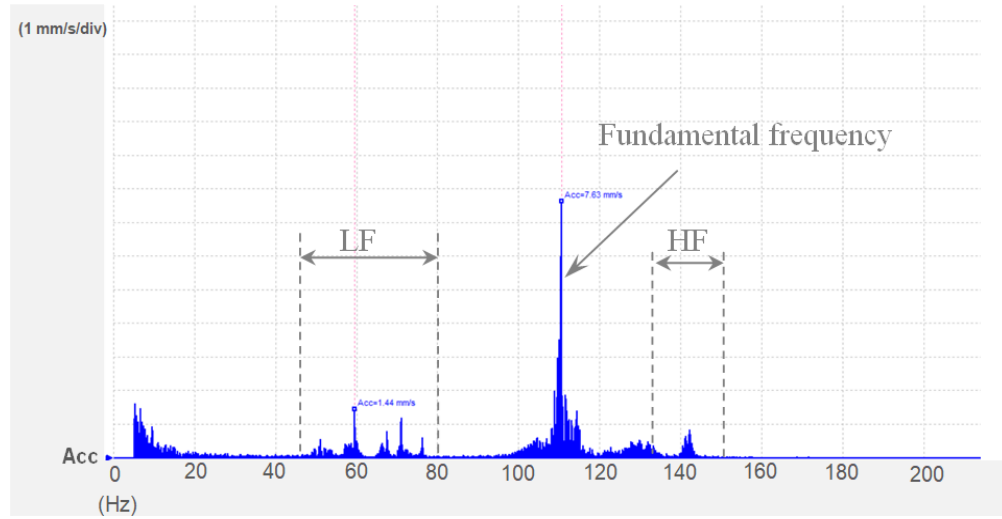

Fig. 9. Spectrum frequency in self excited case. 


\section{Conclusions}

The evolution of science and additive manufacturing have driven the progress of assistive technology, the development of sophisticated medical investigation and treatment for disabled patients, but they have also brought about an array of assistive devices which address to their problems. After optimizing the designed orthosis, the biomechanical analysis of the hand before and after using this device is desired. The orthosis characterization is focused on qualitative analysis, highlighting the frequency range generated by the dynamic's assembly between micro-motors and the orthosis structure. The current research is concerned with the dynamic analysis of the orthotics in terms of a fixed generator frequency, following closely the analysis at variable frequencies. After analysis the orthosis structure is stable, without major variations and with good repeatability. The dynamic operation of the orthosis is useful around the fundamental frequency but with the frequency variation it can provide reactions in different operating ranges and levels.

Changing the frequency and amplitude conditions can also generate episodes of fatigue that may in time generate cracks in the orthosis structure and for this reason a deferred study of behaviour must be achieved. The new studies will consider the numerical and experimental analysis of the orthosis in extreme conditions at the limit of functioning.

The tests on orthosis will be intensified in the future, using different conditions and variations of vibration parameters to obtain a proper response. The precision of angles measurement is important for the intervention strategy and was possible with fewer costs using special program. It is an inexpensive, reliable and can provide enough information to understand the mechanisms of distribution or transfer of intrinsic and extrinsic forces acting on the muscles and ligaments system. Based on this evaluation, the rehabilitation programs can be oriented more to prevent instead to restore.

The advantages of orthosis proposed in this paper are to be used not only in medical recovery centres but also at home. With the new laser measurements technology, design and manufacturing technologies- rapid prototyping, specific orthotics can be made, individual ones that can be equipped with the vibration solution presented.

\section{References}

1. I. Uher, A. Pasterczyk, M.Bigosinska, M. Svedova, Biomedical Journal of Scientific \& Technical Research, 1-4 (2018)

2. C. Bosco, Acta Physiol Scand 124 (4), 507-513 (1985)

3. S.Tyson, R. Kent, NeuroRehabilitation, 28(1), 29-36 (2011)

4. C. Mohora, D. Boboc, C. Dogariu, S.M. Croitoru, D. Anania, D.Tilina, I. Paraschiv Echipamente pentru terapii assistive (Equipments for assistive therapies), Editura Printech, 285 (2014)

5. G. Raveica, C. Mohora, I. Raveica, Ghe. Militaru, Book Series: Balkan Region Conference on Engineering Education \& MSE, 18-21, 153-156 (2012)

6. I. Raveica, C. Mohora, G. Raveica, A. Pena., Applied Mechanics and Materials, 436 277-284 (2013)

7. G.F. Shannon, Medical and biological engineering, 14, 289-294 (1976)

8. K. Homma, S. Hashino, T. Arai, IEEE/ RSJ International Conference on Intelligent Robots and Systems, Innovations and Theory, Practice and Applications (1998)

9. D. Alquacil, H. Pedrero, R. Molina, R. Cano de la Cuerda, Neurologia, 3, 145-153 (2012)

10. H. Rongying, Z. Hongguang, X. Qiang, J Biomim Biomater Tissue Eng., 13, 55-68 (2012)

11. E. Iconaru, M. Ciucurel, M. M., Georgescu, C. Ciucurel, C., BMC Geriatrics, 18, 296 (2018) 